\title{
UV-Mediated Photofunctionalization of Indirect Restorative Materials Enhances Bonding to a Resin-Based Luting Agent
}

\author{
Kyoko Ishikawa, ${ }^{1}$ Monica Yamauti, ${ }^{1,2}$ Antonin Tichy, ${ }^{3}$ Masaomi Ikeda, ${ }^{1,4}$ Takeshi Ueno, ${ }^{5}$ \\ Noriyuki Wakabayashi, ${ }^{5}$ Ornnicha Thanatvarakorn, ${ }^{6}$ Taweesak Prasansuttiporn, ${ }^{7}$ \\ Celso Afonso Klein-Junior, ${ }^{8}$ Akifumi Takahashi, ${ }^{9}$ Tomohiro Takagaki, ${ }^{10}$ \\ Masatoshi Nakajima, ${ }^{1}$ Junji Tagami, ${ }^{1}$ and Keiichi Hosaka $\mathbb{D}^{1,11}$
}

\begin{abstract}
${ }^{1}$ Department of Cariology and Operative Dentistry, Graduate School of Medical and Dental Sciences, Tokyo Medical and Dental University, 1-5-45, Yushima, Bunkyo-ku, Tokyo 113-8549, Japan

${ }^{2}$ Department of Restorative Dentistry, Graduate School of Dental Medicine, Hokkaido University, Kita 13, Nishi 7, Sapporo 001-0012, Japan

${ }^{3}$ Institute of Dental Medicine, First Faculty of Medicine of the Charles University and General University Hospital in Prague, Karlovo namesti 32, Prague 121 11, Czech Republic

${ }^{4}$ Oral Prosthetic Engineering, Graduate School of Medical and Dental Sciences and Technology, Tokyo Medical and Dental University, 1-5-45, Yushima, Bunkyo-ku, Tokyo 113-8549, Japan

${ }^{5}$ Department of Removable Partial Prosthodontics, Graduate School of Medical and Dental Sciences, Tokyo Medical and Dental University, 1-5-45, Yushima, Bunkyo-ku, Tokyo 113-8549, Japan

${ }^{6}$ Faculty of Dentistry, Bangkok Thonburi University, 16/10 Taweewatana, Bangkok 10170, Thailand

${ }^{7}$ Department of Restorative Dentistry and Periodontology, Faculty of Dentistry, Chiang Mai University, Center of Excellence in Materials Science and Technology, Chiang Mai University, T. Suthep, A. Muang, Chiang Mai 50200, Thailand

${ }^{8}$ Department of Operative Dentistry, Lutheran University of Brazil, 301, Cachoeira do Sul, Canoas, RS 96501-595, Brazil

${ }^{9}$ General Dentistry 1, The Nippon Dental University Hospital, 2-3-16 Fujimi, Chiyoda-ku, Tokyo 102-8158, Japan

${ }^{10}$ Department of Operative Dentistry, Division of Oral Functional Science and Rehabilitation, School of Dentistry, Asahi University, 1851 Hozumi, Mizuho, Gifu 501-0296, Japan

${ }^{11}$ Department of Regenerative Dental Medicine, Tokushima University Graduate School of Biomedical Sciences, 3-1815 Kuramotocho, Tokushima 770-8504, Japan
\end{abstract}

Correspondence should be addressed to Keiichi Hosaka; keiichihosaka@gmail.com

Received 18 March 2021; Revised 9 April 2021; Accepted 12 April 2021; Published 30 May 2021

Academic Editor: Turki Bakhsh

Copyright (C) 2021 Kyoko Ishikawa et al. This is an open access article distributed under the Creative Commons Attribution License, which permits unrestricted use, distribution, and reproduction in any medium, provided the original work is properly cited.

\footnotetext{
Purpose. The potential of UV-mediated photofunctionalization to enhance the resin-based luting agent bonding performance to aged materials was investigated. Methods. Sixty samples of each material were prepared. Yttria-stabilized zirconia (YZr) and Pd$\mathrm{Au}$ alloy $(\mathrm{Pd}-\mathrm{Au})$ plates were fabricated and sandblasted. Lithium disilicate glass-ceramic (LDS) was CAD-CAM prepared and ground with \#800 SiC paper. Half of the specimens were immersed in machine oil for $24 \mathrm{~h}$ to simulate the carbon adsorption. Then, all of the specimens (noncarbon- and carbon-adsorbed) were submitted to UV-mediated photofunctionalization with a $15 \mathrm{~W}$ UV-LED $\left(265 \mathrm{~nm}, 300 \mathrm{~mA}, 7692 \mu \mathrm{W} / \mathrm{cm}^{2}\right)$ for 0 (control groups), 5, and $15 \mathrm{~min}$ and subjected to contact angle $(\Theta)$ measurement and bonded using a resin cement (Panavia ${ }^{\mathrm{TM}}$ V5, Kuraray Noritake, Japan). The tensile bond strength (TBS) test was performed after $24 \mathrm{~h}$. The $\Theta\left({ }^{\circ}\right)$ and TBS $(\mathrm{MPa})$ data were statistically analyzed using two-way ANOVA and Bonferroni correction tests $(\alpha=0.05)$. Results. In the carbon-adsorbed groups, UV-mediated photofunctionalization for 5 min significantly decreased $\Theta$ of all materials and increased TBS of YZr, and UV for $15 \mathrm{~min}$ significantly increased the TBS of LDS and Pd-Au. In noncarbon-adsorbed groups, UV-photofunctionalization did not significantly change the $\Theta$ or TBS except YZr specimens UVphotofunctionalized for $15 \mathrm{~min}$. Conclusion. UV-mediated photofunctionalization might have removed the adsorbed
} 
hydrocarbon molecules from the materials' surfaces and enhanced bond strengths of Panavia ${ }^{\mathrm{TM}}$ V5 to YZr, LDS, and Pd-Au. Additionally, UV-mediated photofunctionalization improved the overall TBS of YZr. Further investigation on the optimum conditions of UV photofunctionalization on indirect restorative materials should be conducted.

\section{Introduction}

In recent years, indirect tooth-colored restorative materials such as zirconia and glass-ceramics have been widely used due to their esthetics and increased patients' demand for natural color restorations. Still, in some clinical cases, restorations fabricated with precious metal alloys are necessary. Moreover, based on the concept of minimal intervention dentistry, the development of restorations with higher mechanical strength, better esthetics, and improved bonding performance of resin-based luting agents has enabled minimum tooth preparation placement [1].

The effect of aging, that is, the effect of time after processing the titanium surface, is crucial to the implants' osteoconductivity. Titanium surfaces adsorb hydrocarbons progressively overtime under ambient conditions, which in part determines titanium's surface energy [2]. The atmosphere also modifies other metals and ceramics' exposed surfaces by producing this carbon-containing layer [3-6]. Hydrocarbon molecules' production is unavoidable due to the constant accumulation of carbonyl moiety, particular hydrocarbons from the atmosphere and surrounding environment during the implants' preparation and storage, and indirect restorations placement [7].

When the indirect dental restorative materials are kept and stored in the atmosphere, water and organic molecules which contain carbon are adsorbed on their surface [3-6]. Hydrocarbons can also be adsorbed on zirconia surfaces, leading to increasing contact angle due to the adsorbed hydrocarbons' lower surface energy [8]. For nonpolar chemical species without nonbonding electron pairs such as alkanes $\left(\mathrm{C}_{n} \mathrm{H}_{2 n+2}\right)$, physical carbon adsorption on the zirconia surface has been observed [9]. Furthermore, $\mathrm{CO}_{2}$ is most commonly adsorbed onto zirconium oxide (or zirconia) as the carbonate form [10]. Therefore, as it occurs in implant surfaces, there might be a risk that the cleaned restorative surfaces may be aged from the laboratory's bench to the dental office, which may compromise the bonding performance of the surfaces to a resin-based luting agent, leading to future degradation of restorationluting agent dentin. This speculation and the lack of data to support it led to the present investigation.

The ultraviolet (UV) photofunctionalization was initially used in titanium surfaces to control the biological and timerelated aging from the processing or manufacture $[2,11$, 12] to the implants' use. The UV photofunctionalization aims to decompose and remove the hydrocarbon molecules and restore the titanium's surface hydrophilicity under appropriate conditions, enhancing bone-titanium integration [7, 12-14]. More recently, the UV-mediated photofunctionalization has also been applied to orthodontic miniscrews $[15,16]$, zirconia oxide implants [17-20], gold [6], and other metal alloys as CoCrPd alloy [5] to promote hydrocarbons removal, increase the surface free energy, and stimulate the biointegration and osteoconductivity [17].
However, to the best of our knowledge, few studies have investigated the photofunctioning of aged indirect restorative materials mediated by UV irradiation from the viewpoint of adhesive bonding performance. Therefore, the study evaluated the bond strength of a resin-based luting agent to zirconia, lithium disilicate ceramics, and a Pd-Au alloy after UVmediated photofunctionalization. The feasibility of using a new method to adsorb carbon to surfaces and a prototype of a UV-LED unit was also investigated. The null hypotheses were that UV photofunctionalization of the indirect restorative materials would not affect (1) the contact angle of the surfaces and (2) the tensile bond strength to a resin-based luting agent.

\section{Materials and Methods}

2.1. Study Design and Materials. This was a prospective laboratory study in which different materials were evaluated separately. The independent variables were surface contamination (noncarbon-adsorbed or carbon-adsorbed) and UV-light photofunctionalization time $(0,5$, and $15 \mathrm{~min})$. The outcomes were contact angle and tensile bond strength.

The materials, their basic composition, and manufacturers' instructions used in the study are described in Table 1. The used restorative materials were yttria-stabilized zirconia ceramic (YZr; TZ-3Y-E; Tosoh, Tokyo, Japan), lithium disilicate ceramic (LDS; IPS E-max CAD; Ivoclar Vivadent, Schaan, Liechtenstein), and a $\mathrm{Pd}-\mathrm{Au}$ alloy (Pd-Au, Castmaster 12S; IDS, Tokyo, Japan).

2.2. Specimen Preparation. For each restorative material, sixty specimens, custom-made plates $(10 \times 10 \times 2 \mathrm{~mm})$, were prepared in a dental laboratory. After YZr sintering, LDS glazing, and $\mathrm{Pd}-\mathrm{Au}$ casting, the specimens were used in two weeks. Before the specimen preparation, the adherent surfaces of $\mathrm{YZr}$ and $\mathrm{Pd}-\mathrm{Au}$ surfaces were air-abraded with $50 \mu \mathrm{m} \mathrm{Al} \mathrm{Al}_{2} \mathrm{O}_{3}$ particles from a $10 \mathrm{~mm}$ distance for $20 \mathrm{~s}$ at $0.2 \mathrm{MPa}$ and $0.4 \mathrm{MPa}$ pressure respectively, using a blasting machine (Basic Master, Renfert, Hilzingen, Germany), and those of LDS were ground with 800-grit SiC paper (Sankyo Fuji Star, Saitama, Japan) under running tap water. Subsequently, all restorative materials were ultrasonically cleaned in distilled water for $5 \mathrm{~min}$ and dried with oil-free compressed air. Half of the specimens of each material were submitted to carbon adsorption simulation by storing them in a device containing oil composed of various types of hydrocarbons (CxHy) (Yoshida Spray, The Yoshida Dental MFG. Co., LTD., Tokyo, Japan) for an additional period of $24 \mathrm{~h}$, dried with a dental threeway syringe, and were referred as carbon-adsorbed groups. All the specimens were submitted to UV-mediated photofunctionalization using a portable $15 \mathrm{~W}$ UV-LED light generator prototype (UV LED light, $265 \mathrm{~nm}, 300 \mathrm{~mA}, 7692 \mu \mathrm{W} / \mathrm{cm}^{2}$, Nikkiso, Tokyo, Japan) at $1 \mathrm{~cm}$ for 0 (control groups), 5, and $15 \mathrm{~min}$. Those groups that were not previously submitted to 
TABLE 1: Materials' type, name (abbreviation), manufacturer, elemental composition, and instructions of use.

\begin{tabular}{lcc}
\hline Material type, name, (abbreviation), manufacturer & Basic composition & Instructions of use \\
\hline $\begin{array}{l}\text { Yttria-stabilized zirconia ceramic, TZ-3Y-E, } \\
\text { (YZr), Tosoh, Tokyo, Japan }\end{array}$ & $\mathrm{ZrO}_{2}, \mathrm{Y}_{2} \mathrm{O}_{3}, \mathrm{HfO}_{2}, \mathrm{Al}_{2} \mathrm{O}_{3}$ & - \\
\hline
\end{tabular}

Lithium disilicate ceramics, IPS e.max CAD (LDS), $\mathrm{SiO}_{2}, \mathrm{Li}_{2} \mathrm{O}, \mathrm{K}_{2} \mathrm{O}, \mathrm{P}_{2} \mathrm{O}_{5}, \mathrm{ZrO}_{2}, \mathrm{ZnO}_{2} \mathrm{Al}_{2} \mathrm{O}_{3}$, Ivoclar Vivadent, Schaan, Liechtenstein $\mathrm{MgO}$, coloring oxides

Precious metal alloy, Castmaster 12S, (Pd-Au), IDS, Tokyo, Japan

Au 12\%, Pd 20\%, Ag 54\%, Cu 12\%, additives

$>90 \%$ acetone, $10-\mathrm{MDP}$

Paste A: Bis-GMA, TEGDMA, hydrophobic aromatic dimethacrylate, hydrophilic aliphatic dimethacrylate, initiators, accelerators, silanated barium glass filler, silanated aluminum oxide filler, colloidal silica

Dual-cure resin luting agent, Panavia ${ }^{\mathrm{TM}}$ V5, (PV5), Kuraray Noritake, Japan

Paste B: Bis-GMA, hydrophobic aromatic dimethacrylate, hydrophilic aliphatic dimethacrylate, initiators, accelerators, silanated barium glass filler, silanated aluminum oxide filler, accelerators, $\mathrm{dl}$ camphorquinone, pigments
Roughen, wash with water, and dry the metal surface

Apply alloy primer to the metal with a sponge and leave it for drying

Prepare the tooth surface, core, or abutment

Cement with Panavia ${ }^{\mathrm{TM}}$ V5 resin cement

Attach a mixing tip or an endo tip to the syringe of Panavia V5 paste in the usual manner.

Mix the pastes and apply the mixture over the entire adherent surface and place the metal device.

Remove any excess cement

Nontranslucent materials: allow the cement to chemical cure by letting it set for 3 min after placement of the prosthetic restoration.

Translucent materials: light cure the entire surface of the prosthetic restoration using the dental curing unit. If the area to light cure is larger than the light-emitting tip, divide the exposure process into a few applications.

(1) Prepare the adherent (metal, zirconia, glass-ceramic, resin)

(2) Dispense the necessary amount of

Clearfil ${ }^{\mathrm{TM}}$ Ceramic Primer Plus, Kuraray Noritake, Japan

10-MDP, $\gamma$-MTPS, ethanol
Clearfil Ceramic Primer Plus into a well of the mixing dish immediately before application

(3) Dry the entire adherent surface sufficiently using mild, oil-free airflow

$\mathrm{ZrO}_{2}$ : zirconium dioxide; $\mathrm{Y}_{2} \mathrm{O}_{3}$ : yttrium oxide; $\mathrm{HfO}_{2}$ : hafnium oxide; $\mathrm{Al}_{2} \mathrm{O}_{3}$ : aluminum oxide; $\mathrm{SiO}_{2}$ : silicon dioxide; $\mathrm{Li}_{2} \mathrm{O}$ : lithium oxide; $\mathrm{K}_{2} \mathrm{O}$ : potassium oxide; $\mathrm{P}_{2} \mathrm{O}_{5}$ : phosphorus pentoxide; $\mathrm{ZnO}$ : zinc oxide; $\mathrm{MgO}$ : magnesium oxide; Au: gold; Pd: palladium; Ag: silver; Cu: copper; VBATDT: 6-(4)vinyl benzyl-npropyl)amino-1,3,5-trizaine-2,4-dithiol; 10-MDP: 10-methacryloyloxydecyl dihydrogen phosphate; Bis-GMA: bisphenol A-glycidyl methacrylate; TEGDMA: triethylene glycol dimethacrylate; $\boldsymbol{\gamma}$-MTPS: $\boldsymbol{\gamma}$-methacryloxypropyl trimethoxysilane.

carbon adsorption simulation were named noncarbonadsorbed groups after being UV-mediated photofunctionalized.

2.3. Contact Angle ( $\Theta)$ Measurement. The surfaces of the specimens were prepared as described above. A standardized droplet of deionized water $(1 \mu \mathrm{L})$ was placed on the surface of each materials' specimen, and the contact angle $(\theta)$ was measured using a video contact angle system (VCA OPTIMA XE, AST Products. Inc., MA, USA) at room temperature $25^{\circ} \mathrm{C}$ and humidity $50 \pm 10 \%$. The droplet's surface was monitored continuously, and the contact angle was measured just after $20 \mathrm{~s}$ when the droplet was stabilized [21]. Each measurement $\left({ }^{\circ}\right)$ was taken in sextuplicate for each specimen $(n=20)$.
2.4. Tensile Bond Strength (TSB) Test. After the contact angle measurements, the surfaces of the materials were covered with a polyethylene film (100 $\mu$ m thickness) with a circular hole (diameter $4 \mathrm{~mm}$ ), in which the Ceramic Primer Plus (Kuraray Noritake Dental Corp., Japan) and the resin-based luting agent (Panavia ${ }^{\mathrm{TM}}$ V5, Kuraray Noritake Dental Corp., Japan) were applied as per the manufacturer's instructions. A stainless steel metal rod previously sandblasted and treated with a metal primer (Alloy Primer, Kuraray Noritake Dental Corp.) was carefully and vertically pressed against the mixed luting agent, and excess of the material was removed off using a micro brush. The resin-based luting agent was light cured for $10 \mathrm{~s}$ from four sides using an LED light-curing unit (Valo 
Grande, $1200 \mathrm{~mW} / \mathrm{cm}^{2}$, Ultradent, UT, USA). After the 30 min setting time, all specimens were stored in distilled water at $37^{\circ} \mathrm{C}$ for $24 \mathrm{~h}$. After storage, the specimens were positioned in a testing apparatus with the bonded interface oriented perpendicularly to the tensile load and subjected to the TBS test at a crosshead speed of $0.5 \mathrm{~mm} / \mathrm{min}$ (Autograph AGS-J, Shimadzu, Kyoto, Japan). After testing, the debonded interfaces were observed using a stereomicroscope (Nikon SMZ1000, Nikon, Tokyo, Japan) at a 35x magnification for failure mode classification. Failure modes were classified into three following categories: (1) adhesive failure at the interface between the materials and the resin cement, (2) cohesive failure within the resin cement, and (3) mixed failure combining adhesive and cohesive failures [21].

2.5. Statistical Analysis. The TBS and $\theta$ data of each indirect restorative material were tested for normality and homoscedasticity. As the values followed a normal and homogeneous distribution, the data of both outcomes were statistically analyzed using a two-way ANOVA for each material (carbon - adsorption $\times$ duration of UV-mediated photofunctionalization), followed by a $t$-test with Bonferroni correction for pairwise comparisons. No comparisons between the materials were performed as this was not part of the research question. The analyses were performed at a significance level of 0.05 using the IBM SPSS Statistics 27 software (IBM Corp., Armonk, NY, USA).

\section{Results}

3.1. Contact Angle. The mean $\theta$ and standard deviations of YZr, LDS, and Pd-Ag are presented in Table 2. Two-way ANOVA revealed that $\theta$ values were significantly affected by carbon adsorption and UV photofunctionalization time as per each restorative material. There was a significant interaction between carbon adsorption and each material's UV application time. For all materials, in the carbon-adsorbed groups, the $\Theta$ significantly decreased as the UV application time increased. On the contrary, there were no significant differences among UV application time in the noncarbonadsorbed groups of all restorative materials. For all materials, in the control groups (no UV application), the carbonadsorbed groups presented higher $\theta$ values than those of noncarbon-adsorbed groups.

For each material, different superscript letters indicate significant differences between groups: uppercase letters in a column and lowercase letters in the row; ${ }^{*} x^{* *}$ interaction between variables.

3.2. TBS. The mean TBS and standard deviations of Panavia $^{\mathrm{TM}}$ V5 to UV-photofunctionalized YZr, LDS, and Pd-Au specimens are presented in Table 3. Two-way ANOVA revealed that UV-mediated photofunctionalization and application time significantly affected TBS for all materials. Moreover, there was a significant interaction between the independent variables in the case of LDS and Pd-Au. For noncarbon-adsorbed groups, UV-mediated functionalization for $15 \mathrm{~min}(35.2 \pm 7.7 \mathrm{MPa})$ had a positive effect on the bond strength of YZr to Panavia ${ }^{\mathrm{TM}}$ V5 but did not improve the bonding of the other materials. On the other hand, the UV photofunctionalization significantly increased the TBS of carbon-adsorbed groups of all materials to the luting agent, mainly when $15 \mathrm{~min}$ application was performed. In the control groups (i.e., no UV-mediated photofunctionalization), the carbon adsorption harmed the bond strength of all materials to the resin-based luting agent, indicating the effect of the oil-storage method.

For each material, different superscript letters indicate significant differences between groups $(p<0.05)$ : uppercase letters in a column and lowercase letters in a row; ${ }^{*} x^{* *}$ interaction between variables.

3.3. Failure Mode Analysis. Failure mode distributions of debonded specimens are shown in Figure 1. In the $\mathrm{YZr}$ specimens, the predominant failure mode was adhesive (interfacial) in carbon-adsorption groups, while mixed failure prevailed in the noncarbon-adsorbed groups. The extended UV-mediated photofunctionalization treatment decreased interfacial failures and increased both cohesive and mixed failures in both noncarbon-adsorbed and carbon-adsorbed groups. For LDS and Pd-Au, the predominant failure mode was adhesive (interfacial) failure in noncarbon-adsorbed and carbonadsorbed groups. In carbon-adsorbed groups, neither cohesive nor mixed failures were observed except in 15 min UV mediate photofunctionalization noncarbon-adsorbed groups.

\section{Discussion}

The adsorption of carbon has been a recurrent issue in implantology and orthodontics as the surfaces of titanium implants, and orthodontic miniscrews suffer contamination with hydrocarbons, compromising their bioinductivity, cellular migration, and osteointegration [7, 22]. Moreover, the fixation of indirect materials to tooth substrates requires luting agents, being the resin-based ones widely used [23]. The resin-based luting agents require restorations and tooth substrates' physicochemical preparation and cleaning that are restorative- and luting agent-dependent [24]. The preparation of restorations' inner surfaces is a critical step in which high pressurized particle abrasion or strong acids (i.e., hydrofluoric acid-HF) can be used [23, 24]. However, in Japan, hydrofluoric acid cannot be used by dental clinicians since a 3-year-old child patient died of acute drug intoxication from hydrofluoric acid in 1982 when fluoride solution was misplaced by the acid [25]. Thus, nowadays, dentists cannot buy $\mathrm{HF}$ acid, and they rely on the dental technician for the HF application. Alternatively, the dentist would use phosphoric acid to etch before the silane coating is applied [25-27]. More recently, a hypersaturated solution of zirconium oxide particles in sodium hydroxide and water has been introduced and showed an effective decontamination potential to clean zirconium surface by removing its organic contents [28-30]). Still, there is a continuous search for alternatives to replace HF clinical practice, especially now that there is an increase in indirect restorations that demands surface pretreatment before luting.

This in vitro study showed that UV-mediated photofunctionalization reduced the contact angle of ceramics and metal, allowing a more appropriate spread of the primers 
TABLE 2: Mean(SD) $\left({ }^{\circ}\right)$ of the contact angle $(\Theta)$ according to the carbon adsorption condition* and UV-mediated photofunctionalization time**.

\begin{tabular}{|c|c|c|c|c|c|}
\hline \multirow{2}{*}{ Material } & \multirow{2}{*}{ Surface condition } & \multicolumn{3}{|c|}{ UV-mediated photofunctionalization time } & \multirow[t]{2}{*}{ Significance $(f ; p)$} \\
\hline & & $0 \mathrm{~min}$ & $5 \mathrm{~min}$ & $15 \mathrm{~min}$ & \\
\hline \multirow[b]{2}{*}{ YZr } & Noncarbon-adsorbed & $34.2(2.4)^{\mathrm{aA}}$ & $32.8(2.8)^{\mathrm{aA}}$ & $34.1(2.4)^{\mathrm{aA}}$ & $* 7400.55 ;<0.001$ \\
\hline & Carbon-adsorbed & $97.9(2.7)^{\mathrm{aB}}$ & $86.8(3.9)^{\mathrm{bB}}$ & $66.1(3.9)^{\mathrm{cB}}$ & $\begin{array}{l}\quad * * 256.27 ;<0.001 \\
{ }^{*} \times^{* *} 261.95 ;<0.001\end{array}$ \\
\hline \multirow[b]{2}{*}{ LDS } & Noncarbon-adsorbed & $5.1(1.3)^{\mathrm{aA}}$ & $5.3(1.3)^{\mathrm{aA}}$ & $5.0(1.5)^{\mathrm{aA}}$ & $* 2732.23 ;<0.001$ \\
\hline & Carbon-adsorbed & $32.3(3.6)^{\mathrm{aB}}$ & $29.1(2.7)^{\mathrm{bB}}$ & $25.1(3.1)^{\mathrm{cB}}$ & $\begin{array}{c}{ }^{* *} 21.70 ;<0.001 \\
{ }^{*} \times^{* *} 19.70 ;<0.001\end{array}$ \\
\hline \multirow[b]{2}{*}{$\mathrm{Pd}-\mathrm{Au}$} & Noncarbon-adsorbed & $57.3(2.3)^{\mathrm{aA}}$ & $57.2(2.4)^{\mathrm{aA}}$ & $54.9(4.1)^{\mathrm{aA}}$ & *1190.10;<0.001 \\
\hline & Carbon-adsorbed & $87.5(4.2)^{\mathrm{aB}}$ & $80.6(4.4)^{\mathrm{bB}}$ & $67.3(2.3)^{\mathrm{cB}}$ & $\begin{array}{c}{ }^{* *} 108.90 ;<0.001 \\
{ }^{*} \times{ }^{* *} 66.66 ;<0.001\end{array}$ \\
\hline
\end{tabular}

TABLE 3: Tensile bond strengths to various substrates: mean(SD) [MPa].

\begin{tabular}{|c|c|c|c|c|c|}
\hline \multirow{2}{*}{ Material } & \multirow{2}{*}{ Surface condition } & \multicolumn{3}{|c|}{ UV-mediated photofunctionalization time } & \multirow{2}{*}{$\begin{array}{c}\text { Significance } \\
(f ; p)\end{array}$} \\
\hline & & $0 \mathrm{~min}$ & $5 \mathrm{~min}$ & $15 \mathrm{~min}$ & \\
\hline \multirow[b]{2}{*}{$\mathrm{YZr}$} & Noncarbon-adsorbed & $25.3(4.5)^{\mathrm{aA}}$ & $29.2(5.7)^{\mathrm{aA}}$ & $35.2(7.7)^{\mathrm{bA}}$ & $* 240.85 ;<0.001$ \\
\hline & Carbon-adsorbed & $9.6(4.1)^{\mathrm{aB}}$ & $14.6(4.7)^{\mathrm{bB}}$ & $18.9(4.5)^{\mathrm{cB}}$ & $\begin{array}{l}{ }^{* *} 30.69 ;<0.001 \\
{ }^{*} \times{ }^{* *} 0.26 ; 0.773\end{array}$ \\
\hline \multirow[b]{2}{*}{ LDS } & Noncarbon-adsorbed & $11.8(3.6)^{\mathrm{aA}}$ & $11.6(3.6)^{\mathrm{aA}}$ & $12.8(4.1)^{\mathrm{aA}}$ & $* 47.99 ;<0.001$ \\
\hline & Carbon-adsorbed & $5.0(2.5)^{\mathrm{aB}}$ & $6.8(3.0)^{\mathrm{aB}}$ & $11.2(3.4)^{\mathrm{bA}}$ & $\begin{array}{l}{ }^{* *} 11.40 ;<0.001 \\
{ }^{*} \times{ }^{* *} 5.56 ; 0.005\end{array}$ \\
\hline \multirow[b]{2}{*}{$\mathrm{Pd}-\mathrm{Au}$} & Noncarbon-adsorbed & $16.7(2.7)^{\mathrm{aA}}$ & $17.0(4.7)^{\mathrm{aA}}$ & $18.5(4.1)^{\mathrm{aA}}$ & $* 210.08 ;<0.001$ \\
\hline & Carbon-adsorbed & $5.1(1.9)^{\mathrm{aB}}$ & $6.7(2.2)^{\mathrm{aB}}$ & $13.1(3.5)^{\mathrm{bA}}$ & $\begin{array}{c}{ }^{* *} 22.74 ;<0.001 \\
{ }^{*} x^{* *} 9.18 ;<0.001\end{array}$ \\
\hline
\end{tabular}

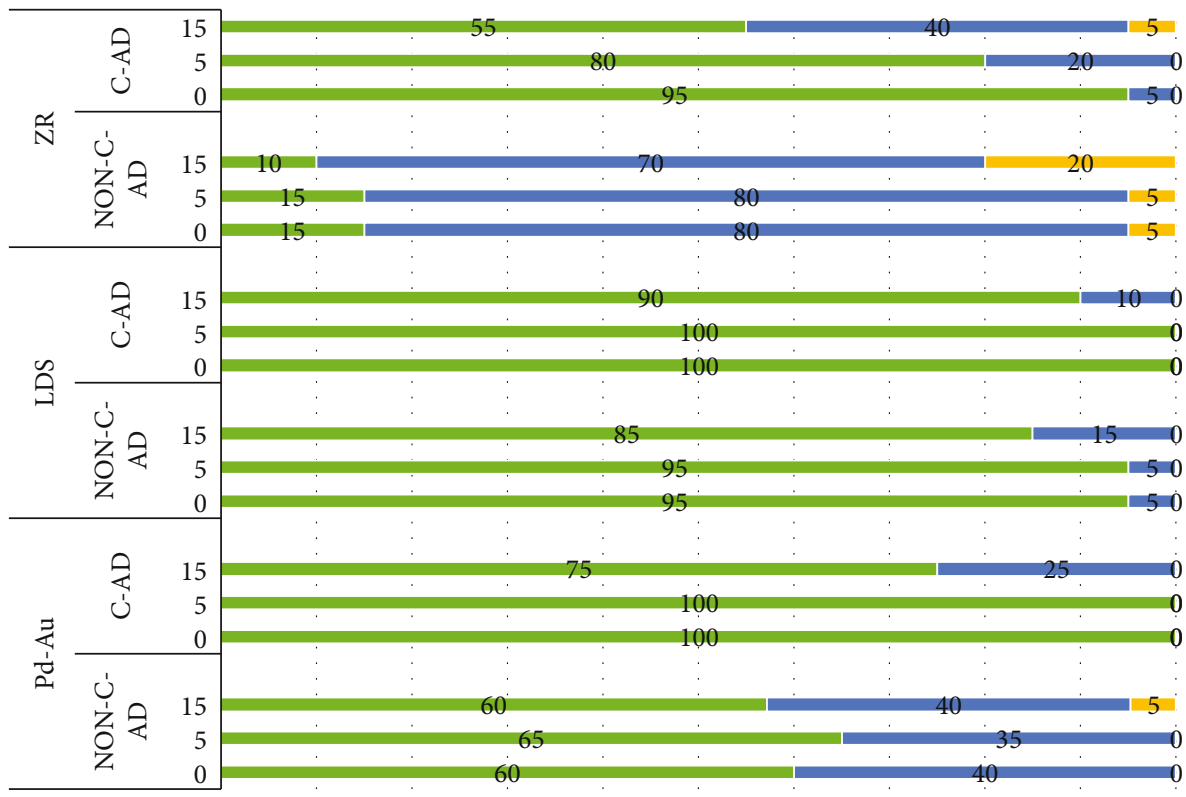

C-AD: carbon-adsorbed; NON-C-AD: non-carbon adsorbed

- Adhesive

- Mixed

Cohesive

FIGURE 1: Failure mode (\%) as per restorative materials and UV-photofunctionalization treatment and time. 
$[31,32]$ and improved their bond strength. From a clinical perspective, the restorations were left two weeks to age (that is, to adsorb carbon naturally), and then the adherent surface was prepared to simulate the clinical situation. Therefore, both null hypotheses were rejected. The UV photofunctionalization might help replace the $\mathrm{HF}$ as a decontaminant agent, as demonstrated in implant and miniscrew surfaces $[16,22$, 33]. Furthermore, due to the indirect restorations' occlusal adjustment and saliva contamination during try-in [34, 35], it is expected that dentists would return the restorations to the dental laboratory for repolishing of outer surfaces [36] and sandblasting of inner surfaces [34-36]. Consequently, an additional bench-chairside time lag could occur, jeopardizing the adherent's surface cleanliness.

YZr benefited the most from the treatment, as even 5minute UV photofunctionalization significantly increased its bond strength. This finding agrees with the previous study reporting that UV treatment to tetragonal zirconia polycrystal (as the one used in this study) remarkably decreased the carbon content and increased the surface's hydrophilicity [37, 38]. Regardless of adherent material, $15 \mathrm{~min}$ of UV-mediated photofunctionalization of the carbon-adsorbed specimens promoted a significant decrease in the $\theta$ (Table 2) and increased bond strength compared to the control groups $(0 \mathrm{~min})$ (Table 3). The contact angle decrease promoted more intimate interaction between the resin-based luting agent and the adherent, favoring the bond strength [37]. The increase of bond strength resulted in mixed and cohesive failures of carbonadsorbed groups (Figure 1). The relation between decreased contact angle followed by increased adherent surface wettability and increased bond strength was extensively investigated $[30,39,40]$.

In noncarbon-adsorbed groups, the influence of UV photofunctionalization on $\theta$ and TBS was not observed in LDS and Pd-Au specimens. However, 15-minute UV treatment significantly increased YZr's TBS with increased cohesive failures; although, $\Theta$ values were not significantly changed. This finding may indicate that UV photofunctionalization could have an additional effect to enhance the YZr's bond strength compared to sandblasting followed by a 10 MDP containing primer only. Due to this effect of UV irradiation [18, 41, 42], stainless steel [43] and fiber posts [44] have improved these materials' bond strength to dentin.

Considering the control groups (non-UV-mediated), regardless of materials, the $\theta$ of noncarbon-adsorbed specimens was lower, and TBS was higher than those results of carbon-adsorbed counterparts. These findings strongly suggested that the oil storage method could artificially adsorb carbon on the adherents' surfaces. Furthermore, the statistical differences between the control and UV photofunctionalized groups indicate that UV-irradiation positively affected those surfaces by removing impurities such as hydrocarbons and increasing their wettability $[41,42,45]$.

The present study's strengths also rely on the proposed oil storage model to accelerate biological aging and the UVmediated photofunctionalization device. A prototype of portable UV-LED equipment was employed in the study, aiming at the chairside or dental laboratory use. However, further research should address the chemical aspects of car- bon adsorption in different indirect materials, using the oil storage model and the effect of the portable UV photofunctionalization. Studies on the optimum conditions (distance, duration of irradiation, power, absence of ozone) of UVmediated photofunctionalization are also needed.

\section{Conclusions}

Within this study's limitation, it is suggested that UVmediated photofunctionalization could decrease the contact angle of YZr, LDS, and Pd-Au surfaces and enhance the bond strengths to a resin-based luting cement.

\section{Data Availability}

The data used to support the findings of this study are included within the article.

\section{Conflicts of Interest}

The authors declare that there is no conflict of interest regarding the publication of this paper.

\section{Acknowledgments}

This work was supported by the Ministry of Education, Culture, Sports, Science, and Technology of Japan (grant numbers $18 \mathrm{~K} 09571$ and 20K09983) and partially supported by Chaing Mai University.

\section{References}

[1] U. Blunck, S. Fischer, J. Hajtó, and R. Frankenberger, "Ceramic laminate veneers: effect of preparation design and ceramic thickness on fracture resistance and marginal quality in vitro," Clinical Oral Investigations, vol. 24, no. 8, pp. 2745-2754, 2020.

[2] N. Hori, W. Att, T. Ueno et al., "Age-dependent degradation of the protein adsorption capacity of titanium," Journal of Dental Research, vol. 88, no. 7, pp. 663-667, 2009.

[3] H. U. Foersterling and K. H. Hallmeier, "Investigations of the adsorption of palladium on carbonaceous adsorbents modified with dimethylglyoxime, III the adsorption of palladium on a lignite in its unmodified form and modified with dimethylglyoxime," Carbon, vol. 28, no. 4, pp. 503-508, 1990.

[4] M. Soleimani and T. Kaghazchi, "Adsorption of gold ions from industrial wastewater using activated carbon derived from hard shell of apricot stones - an agricultural waste," Bioresource Technology, vol. 99, no. 13, pp. 5374-5383, 2008.

[5] M. Morra, C. Cassinelli, G. Bruzzone et al., "Surface chemistry effects of topographic modification of titanium dental implant surfaces: 1. Surface analysis," The International Journal of Oral \& Maxillofacial Implants, vol. 18, no. 1, pp. 40-45, 2003.

[6] R. Hayashi, T. Ueno, S. Migita et al., "Hydrocarbon deposition attenuates osteoblast activity on titanium," Journal of Dental Research, vol. 93, no. 7, pp. 698-703, 2014.

[7] T. Ogawa, "Ultraviolet photofunctionalization of titanium implants," The International Journal of Oral \& Maxillofacial Implants, vol. 29, no. 1, pp. e95-e102, 2014. 
[8] S. Takeda, M. Fukawa, Y. Hayashi, and K. Matsumoto, "Surface $\mathrm{OH}$ group governing adsorption properties of metal oxide films," Thin Solid Films, vol. 339, no. 1-2, pp. 220-224, 1999.

[9] S. Jalili and M. Keshavarz, "Zirconia ( $\left.\begin{array}{lll}1 & 1 & 0\end{array}\right)$ surface adsorption behavior - a density functional theory study," Computational and Theoretical Chemistry, vol. 1173, article 112702, 2020.

[10] P. Boulet, C. Knofel, B. Kuchta, V. Hornebecq, and P. L. Llewellyn, "Computational investigation of the adsorption of carbon dioxide onto zirconium oxide clusters," Journal of Molecular Modeling, vol. 18, no. 11, pp. 4819-4830, 2012.

[11] W. Att, N. Hori, F. Iwasa, M. Yamada, T. Ueno, and T. Ogawa, "The effect of UV-photofunctionalization on the time-related bioactivity of titanium and chromium-cobalt alloys," Biomaterials, vol. 30, no. 26, pp. 4268-4276, 2009.

[12] T. Ueno, M. Yamada, T. Suzuki et al., "Enhancement of bonetitanium integration profile with UV-photofunctionalized titanium in a gap healing model," Biomaterials, vol. 31, no. 7, pp. 1546-1557, 2010.

[13] F. Iwasa, N. Hori, T. Ueno, H. Minamikawa, M. Yamada, and T. Ogawa, "Enhancement of osteoblast adhesion to UVphotofunctionalized titanium via an electrostatic mechanism," Biomaterials, vol. 31, no. 10, pp. 2717-2727, 2010.

[14] M. Lorenzetti, G. Bernardini, T. Luxbacher, A. Santucci, S. Kobe, and S. Novak, "Surface properties of nanocrystalline $\mathrm{TiO} 2$ coatings in relation to the in vitro plasma protein adsorption," Biomedical Materials, vol. 10, no. 4, article 045012, 2015.

[15] M. Tabuchi, T. Ikeda, M. Hirota et al., "Effect of UV photofunctionalization on biologic and anchoring capability of orthodontic miniscrews," The International Journal of Oral \& Maxillofacial Implants, vol. 30, no. 4, pp. 868-879, 2015.

[16] S. H. Choi, J. Shin, J. K. Cha, J. S. Kwon, J. Y. Cha, and C. J. Hwang, "Evaluation of success rate and biomechanical stability of ultraviolet-photofunctionalized miniscrews with short lengths," American Journal of Orthodontics and Dentofacial Orthopedics, vol. 159, no. 2, pp. 158-166, 2021.

[17] M. Roy, A. Pompella, J. Kubacki et al., "Photofunctionalization of dental zirconia oxide: surface modification to improve biointegration preserving crystal stability," Colloids and Surfaces B: Biointerfaces, vol. 156, pp. 194-202, 2017.

[18] A. Henningsen, R. Smeets, R. Heuberger et al., "Changes in surface characteristics of titanium and zirconia after surface treatment with ultraviolet light or non-thermal plasma," European Journal of Oral Sciences, vol. 126, no. 2, pp. 126-134, 2018.

[19] R. Smeets, A. Henningsen, R. Heuberger, O. Hanisch, F. Schwarz, and C. Precht, "Influence of UV irradiation and cold atmospheric pressure plasma on zirconia surfaces: an in vitro study," The International Journal of Oral \& Maxillofacial Implants, vol. 34, no. 2, pp. 329-336, 2019.

[20] S. H. Choi, J. H. Ryu, J. S. Kwon et al., "Effect of wet storage on the bioactivity of ultraviolet light- and non-thermal atmospheric pressure plasma-treated titanium and zirconia implant surfaces," Materials Science and Engineering: C, vol. 105, article 110049, 2019.

[21] A. Ali, T. Takagaki, T. Nikaido, A. Abdou, and J. Tagami, "Influence of ambient air and different surface treatments on the bonding performance of a CAD/CAM composite block," The Journal of Adhesive Dentistry, vol. 20, no. 4, pp. 317324, 2018.
[22] M. Hirota, T. Ozawa, T. Iwai, K. Mitsudo, and T. Ogawa, "UVmediated photofunctionalization of dental implant: a sevenyear results of a prospective study," Journal of Clinical Medicine, vol. 9, no. 9, p. 2733, 2020.

[23] A. P. Manso and R. M. Carvalho, "Dental cements for luting and bonding restorations," Dental Clinics of North America, vol. 61, no. 4, pp. 821-834, 2017.

[24] A. P. Manso, N. R. F. A. Silva, E. A. Bonfante, T. A. Pegoraro, R. A. Dias, and R. M. Carvalho, "Cements and adhesives for all-ceramic restorations," Dental Clinics of North America, vol. 55, no. 2, pp. 311-332, 2011.

[25] A. Kameyama, A. Haruyama, A. Tanaka et al., "Repair bond strength of a resin composite to plasma-treated or UVIrradiated CAD/CAM ceramic surface," Coatings, vol. 8, no. 7, p. 230, 2018.

[26] K. Klosa, S. Wolfart, F. Lehmann, H.-J. Wenz, and M. Kern, "The effect of storage conditions, contamination modes and cleaning procedures on the resin bond strength to lithium disilicate ceramic," The Journal of Adhesive Dentistry, vol. 11, no. 2, pp. 127-135, 2009.

[27] B. Lapinska, J. Rogowski, J. Nowak, J. Nissan, J. Sokolowski, and M. Lukomska-Szymanska, "Effect of surface cleaning regimen on glass ceramic bond strength," Molecules, vol. 24, no. 3, p. 389, 2019.

[28] P. Angkasith, J. O. Burgess, M. C. Bottino, and N. C. Lawson, "Cleaning methods for zirconia following salivary contamination," Journal of Prosthodontics, vol. 25, no. 5, pp. 375-379, 2016.

[29] W. M. Negreiros, G. M. B. Ambrosano, and M. Giannini, "Effect of cleaning agent, primer application and their combination on the bond strength of a resin cement to two yttriumtetragonal zirconia polycrystal zirconia ceramics," European Journal of Dentistry, vol. 11, no. 1, pp. 6-11, 2017.

[30] M. D. S. Noronha, B. M. Fronza, C. B. André et al., "Effect of zirconia decontamination protocols on bond strength and surface wettability," Journal of Esthetic and Restorative Dentistry, vol. 32, no. 5, pp. 521-529, 2020.

[31] H. A. Wege, J. A. Aguilar, M. Á. Rodríguez-Valverde, M. Toledano, R. Osorio, and M. Á. Cabrerizo-Vílchez, "Dynamic contact angle and spreading rate measurements for the characterization of the effect of dentin surface treatments," Journal of Colloid and Interface Science, vol. 263, no. 1, pp. 162-169, 2003.

[32] A. Tsujimoto, M. Iwasa, Y. Shimamura, R. Murayama, T. Takamizawa, and M. Miyazaki, "Enamel bonding of single-step self-etch adhesives: influence of surface energy characteristics," Journal of Dentistry, vol. 38, no. 2, pp. 123130, 2009.

[33] K. Nakhaei, M. Ishijima, T. Ikeda, A. Ghassemi, J. Saruta, and T. Ogawa, "Ultraviolet light treatment of titanium enhances attachment, adhesion, and retention of human oral epithelial cells via decarbonization," Materials, vol. 14, no. 1, p. 151, 2021.

[34] A. Takahashi, T. Takagaki, T. Wada, M. Uo, T. Nikaido, and J. Tagami, "The effect of different cleaning agents on saliva contamination for bonding performance of zirconia ceramics," Dental Materials Journal, vol. 37, no. 5, pp. 734-739, 2018.

[35] B. Yang, H. C. Lange-Jansen, M. Scharnberg et al., "Influence of saliva contamination on zirconia ceramic bonding," Dental Materials, vol. 24, no. 4, pp. 508-513, 2008.

[36] S. Janyavula, N. Lawson, D. Cakir, P. Beck, L. C. Ramp, and J. O. Burgess, "The wear of polished and glazed zirconia 
against enamel," Journal of Prosthetic Dentistry, vol. 109, no. 1, pp. 22-29, 2013.

[37] A. Noro, M. Kaneko, I. Murata, and M. Yoshinari, "Influence of surface topography and surface physicochemistry on wettability of zirconia (tetragonal zirconia polycrystal)," Journal of Biomedical Materials Research Part B Applied Biomaterials, vol. 101B, no. 2, pp. 355-363, 2013.

[38] K. Tabari, S. Hosseinpour, and H. Mohammad-Rahimi, “The impact of plasma treatment of Cercon ${ }^{\circledR}$ zirconia ceramics on adhesion to resin composite cements and surface properties," Journal of Lasers in Medical Sciences, vol. 8, Suppl 1, pp. S56-S61, 2017.

[39] R. F. Carvalho, M. P. Rippe, R. M. Melo, M. A. Bottino, and R. O. A. Souza, "Resin bond strength to zirconia: effects of surface treatments and resin cements," General Dentistry, vol. 67, no. 1, pp. 71-77, 2019.

[40] A. P. Farina, D. Cechin, C. M. P. Vidal, A. A. Leme-Kraus, and A. K. Bedran-Russo, "Removal of water binding proteins from dentin increases the adhesion strength of low-hydrophilicity dental resins," Dental Materials, vol. 36, no. 10, pp. e302e308, 2020.

[41] T. Ueno, T. Ikeda, N. Tsukimura et al., "Novel antioxidant capability of titanium induced by UV light treatment," Biomaterials, vol. 108, pp. 177-186, 2016.

[42] M. Hirota, T. Ikeda, Y. Sugita, M. Ishijima, S. Hirota, and T. Ogawa, "Impaired osteoblastic behavior and function on saliva-contaminated titanium and its restoration by UV treatment," Materials Science \& Engineering, C: Materials for Biological Applications, vol. 100, pp. 165-177, 2019.

[43] S. J. Baeza-Robleto, D. M. Villa-Negrete, R. García-Contreras, R. J. Scougall-Vílchis, L. J. Guadarrama-Quiroz, and N. L. Robles-Bermeo, "Effects of ultraviolet irradiation on the bond strength of a composite resin adhered to stainless steel crowns," Pediatric Dentistry, vol. 35, no. 1, pp. 23-26, 2013.

[44] F. Reza and N. S. Ibrahim, "Effect of ultraviolet light irradiation on bond strength of fiber post: evaluation of surface characteristic and bonded area of fiber post with resin cement," European Journal of Dentistry, vol. 9, no. 1, pp. 74-79, 2015.

[45] F. Rupp, L. Scheideler, N. Olshanska, M. de Wild, M. Wieland, and J. Geis-Gerstorfer, "Enhancing surface free energy and hydrophilicity through chemical modification of microstructured titanium implant surfaces," Journal of Biomedical Materials Research. Part A, vol. 76A, no. 2, pp. 323-334, 2006. 